\title{
THREE YEARS OF ANTENA: WHAT WE HAVE DONE!
}

J. A. BELMONTE , M. M. HERNÁNDEZ,

F. PÉREZ HERNÁNDEZ, I. VIDAL AND T. ROCA CORTÉS

Instituto de Astrofísica de Canarias

38200 La Laguna, Tenerife, Spain

E. MICHEL, M. AUVERGNE, M. CHEVRETON,

M. J. GOUPIL, F. SOUFI AND A. BAGLIN

DASGAL, DAEC, DESPA, Observatoire de Paris-Meudon

92195 Meudon, France

S. FRANDSEN, M. VISKUM , H. KJELDSEN

AND J. CHRISTENSEN-DALSGAARD

Institut for Fysik og Astronomi, Aarhus Universitet

8000 Aarhus C, Denmark

F.X. SCHMIDER AND E. FOSSAT

Département d'Astrophysique, Université de Nice

06108 Nice, France

PH. DELACHE*, J. PROVOST , N. AUDARD

AND G. BERTHOMIEU

Observatoire de la Côte d'Azur

06304 Nice, France

M. PAPARÓ , G. KOVÁCS AND L. SZABADOS

Konkoly Observatory, Hungarian Academy of Sciences

1525 Budapest, Hungary

* Deceased October 1994. The rest of the authors wish to dedicate this paper to the memory of their colleague and one of the fathers of ANTENA, Philippe Delache.

Abstract. The European Union Network ANTENA started to work in October 1993. During these last three years, several collaborative projects have been undertaken. ANTENA has offered a very good opportunity for most of the European people doing asteroseismology to work together. The asteroseismological networks STEPHI and STACC have run within the framework of the project, obtaining fairly good results. New instrumentation has also been developed, such as the Four-Channel Stellar Photometer. 


\section{Introduction}

The main objective of the groups included in the ANTENA network (A New TEchnology Network for Asteroseismology) has been to give a substantial impetus to the incipient discipline of asteroseismology from all points of view: the observational side, where networks such as STEPHI and STACC have been running with several campaigns; the theoretical side, obtaining improvements in the understanding of the interiors of pulsating stars; the technical side, developing instrumentation devoted to seismological purposes such as a new version of the Four-Channel Stellar Photometer; and the human side, giving the opportunity of exchanging information and manpower between the centres involved. In the following pages, we will offer a summary of the most remarkable results reached in the different fields developed within the framework of the ANTENA project.

\section{Observational research: STEPHI and STACC networks}

The observational network STEPHI (STEllar PHotometry International) (Michel et al., 1995) has performed two campaigns on candidates belonging to the Praesepe cluster (BQ and BW Cnc, 1995; BS and BT Cnc, 1996) over the last three years. These new observations have the aim of completing the sample of Praesepe $\delta$ Scuti stars initiated with BN and BU Cnc in 1992 (Belmonte et al., 1994). The Praesepe cluster, with $14 \delta$ Scuti stars known so far, offers one of the best scenarios for performing asteroseismology, taking advantage of the common value of distance, age, and metallicity for all their components (Hernández et al., 1996a). This allows a stronger restriction on the modelling of these stars. Theoretical results derived from these observations will be shown in the following section.

The synthetic spectra of the 8 Praesepe $\delta$ Scuti stars observed more extensively are shown in Fig. 1. Multiperiodic behaviour is observed in most of them, the pattern and range of excited frequencies changing with the evolutionary stage. On the other hand, drastic changes of amplitude in a short period of time, due to mutual interferences between very close peaks $(\sim 0.5 \mu \mathrm{Hz})$, are also detected (Fig. 2 and Fig. 3 ).

The STACC network (Small Telescope Array with CCD Cameras) (Frandsen, 1992) has made important efforts in monitoring not well known open clusters in order to find the most suitable target for a campaign, considering hundred of stars at the same time. One of these surveys was made in the southern hemisphere where the cluster NGC 6134 was chosen for observation, providing promising results despite its faintness (Frandsen et al., 1996). NGC 7245 (Fig. 4) was the second target discovered for a possible future campaign in the northern hemisphere (Frandsen et al., 1995). 

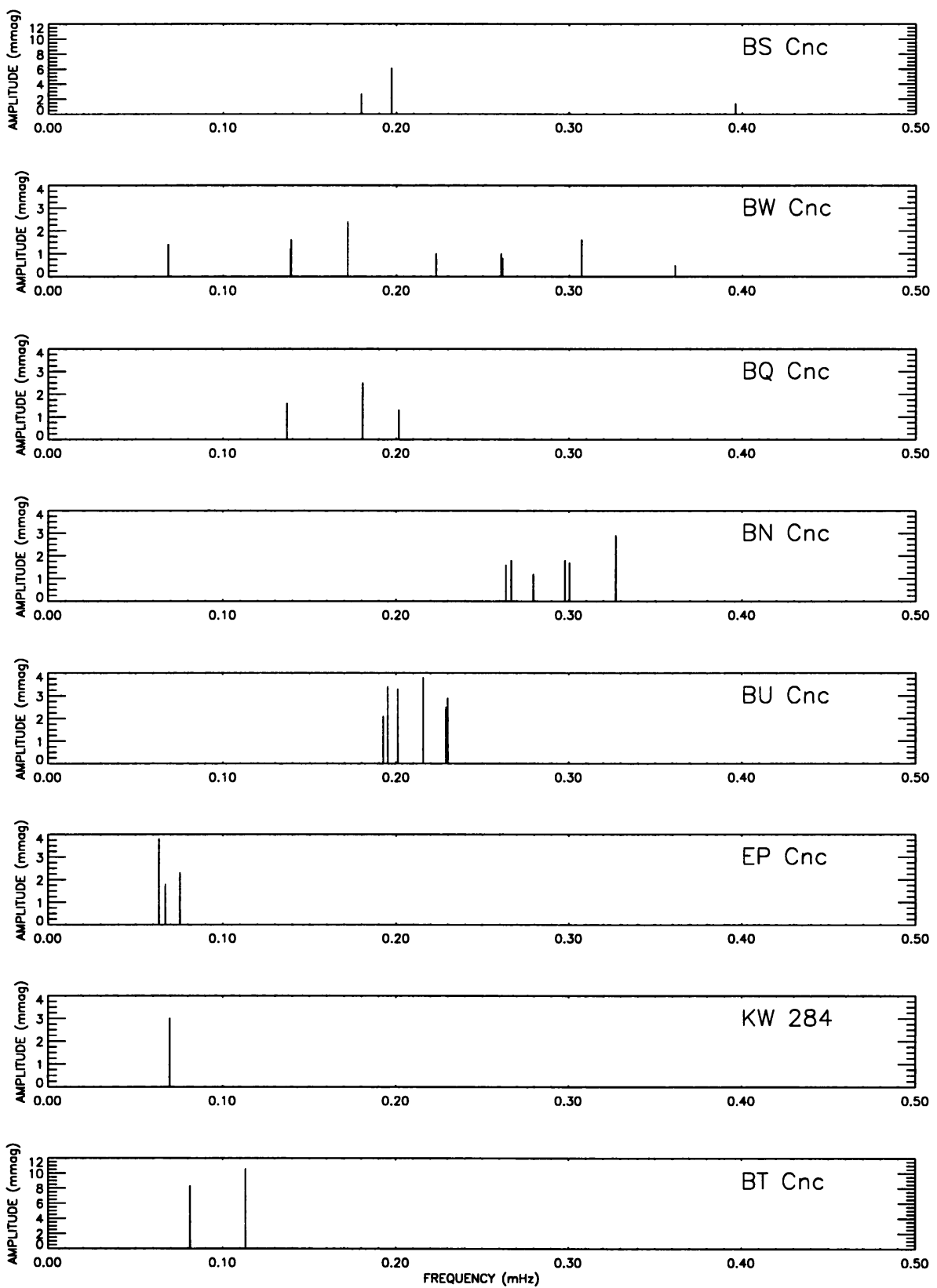

Figure 1. Amplitude spectra of the $8 \delta$ Scuti stars more deeply observed in the Praesepe cluster. They are arranged in increasing luminosity from top to bottom. Detected modes have a confidence level larger than $90 \%$. 

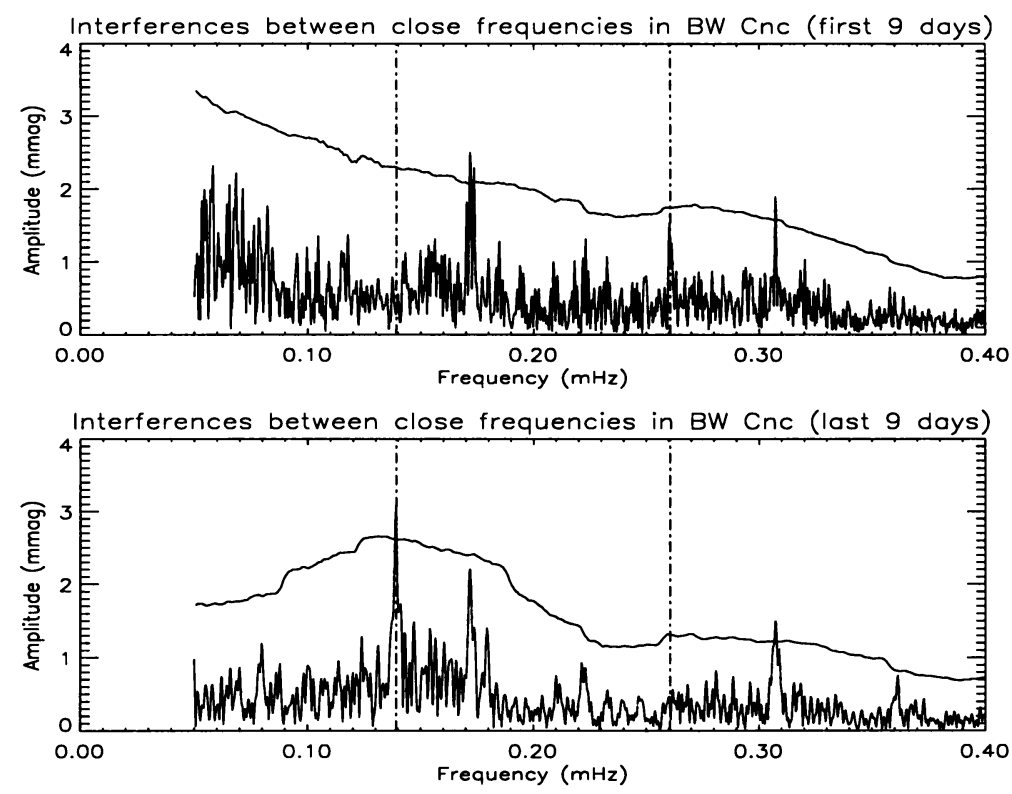

Figure 2. Amplitude change in a short period of time (three weeks, splitted here in two 9-days sets) for the two pairs of close peaks present in BW Cnc. These pairs are only resolved in the whole campaign spectrum. Frequencies of the pairs are indicated with dot-dashed lines. The $99 \%$ confidence level is also plotted (solid line).
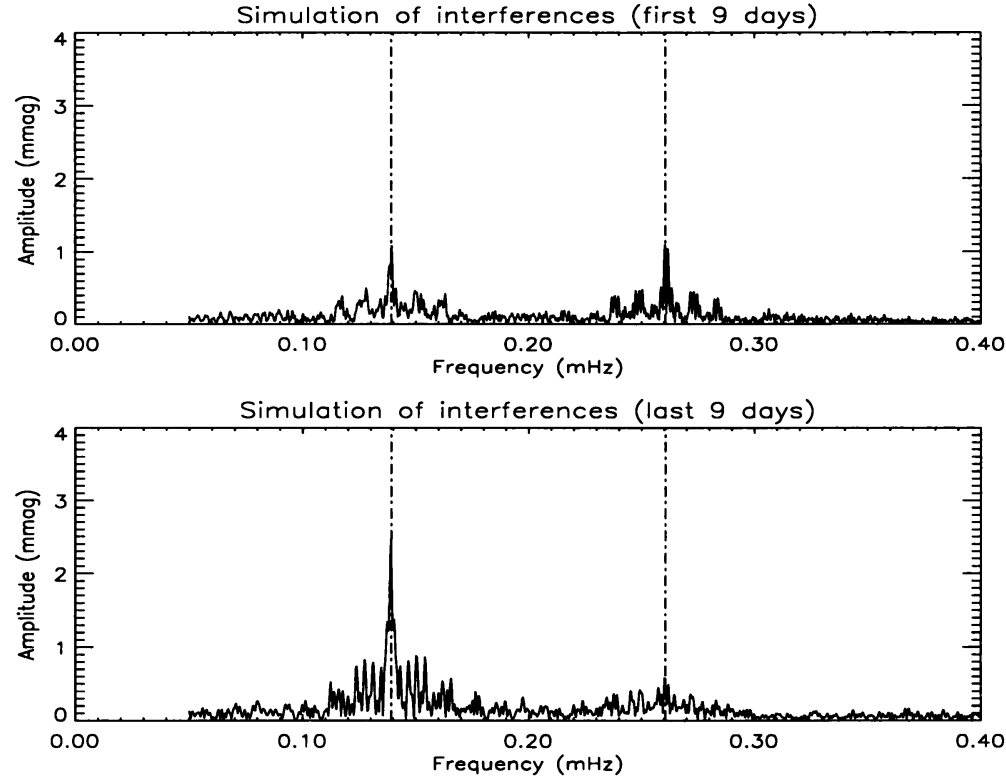

Figure 3. Simulation of the interference for both pairs of close peaks. Frequencies, amplitudes and phases of each peak are taken from the whole campaign spectrum. This shows that the reason of these changes are the mutual interference between the elements of each pair. When facing these situations, studies of secular amplitude variations must be used cautiously since they can be seriously affected by this sort of effect. 


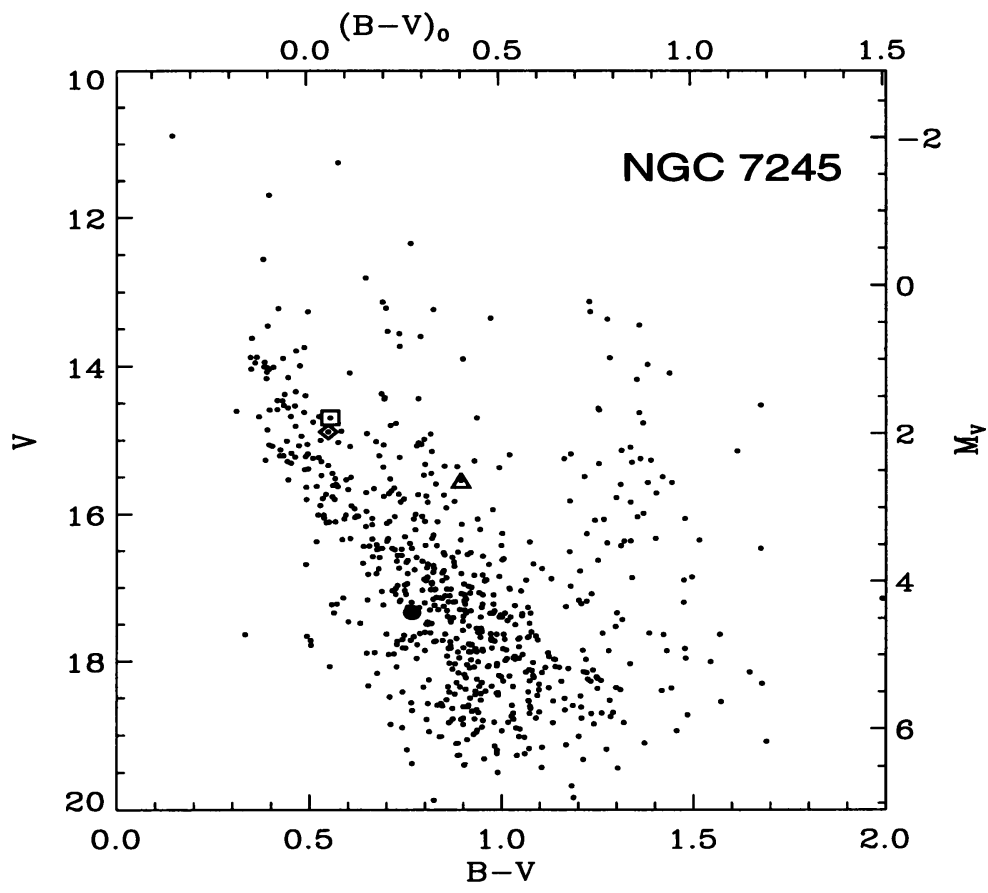

Figure 4. HR diagram for NGC 7245. The four detected variables are indicated. The two stars represented by a square and a diamond are two $\delta$ Scuti stars situated at the hot border of the instability strip. Both stars seem to be multiperiodic with a main period of 0.1167 days and 0.0793 days, respectively. The star represented by a triangle is an eclipsing binary while the star shown as a black circle has a period of 0.2271 days and is probably a W UMa system.

\section{Theoretical results}

The sample of $8 \delta$ Scuti stars monitored extensively in Praesepe has been demonstrated to be extremely useful in terms of restricting the global parameters of the cluster. The ambiguity in the identification of the detected modes in every star separately is now partially removed. The fact of having the same global parameters (age, distance and metallicity) for every member of the cluster, and the little dependence of the ratios of the theoretical radial modes on these parameters, allows a congruent identification of pairs of radial modes in every star for those pairs that can be reproduced with the same parameters for all the stars (Hernández et al., 1996b). In the case of Praesepe, a metallicity close to $Z=0.03$, an age of $640-660 \mathrm{Myr}$ and a distance modulus of 6.4-6.5, match the candidates for radial pulsation frequencies of four cluster members (Fig. 5). In this analysis, an overshooting of $\alpha_{o v}=0.20$ has been considered. 

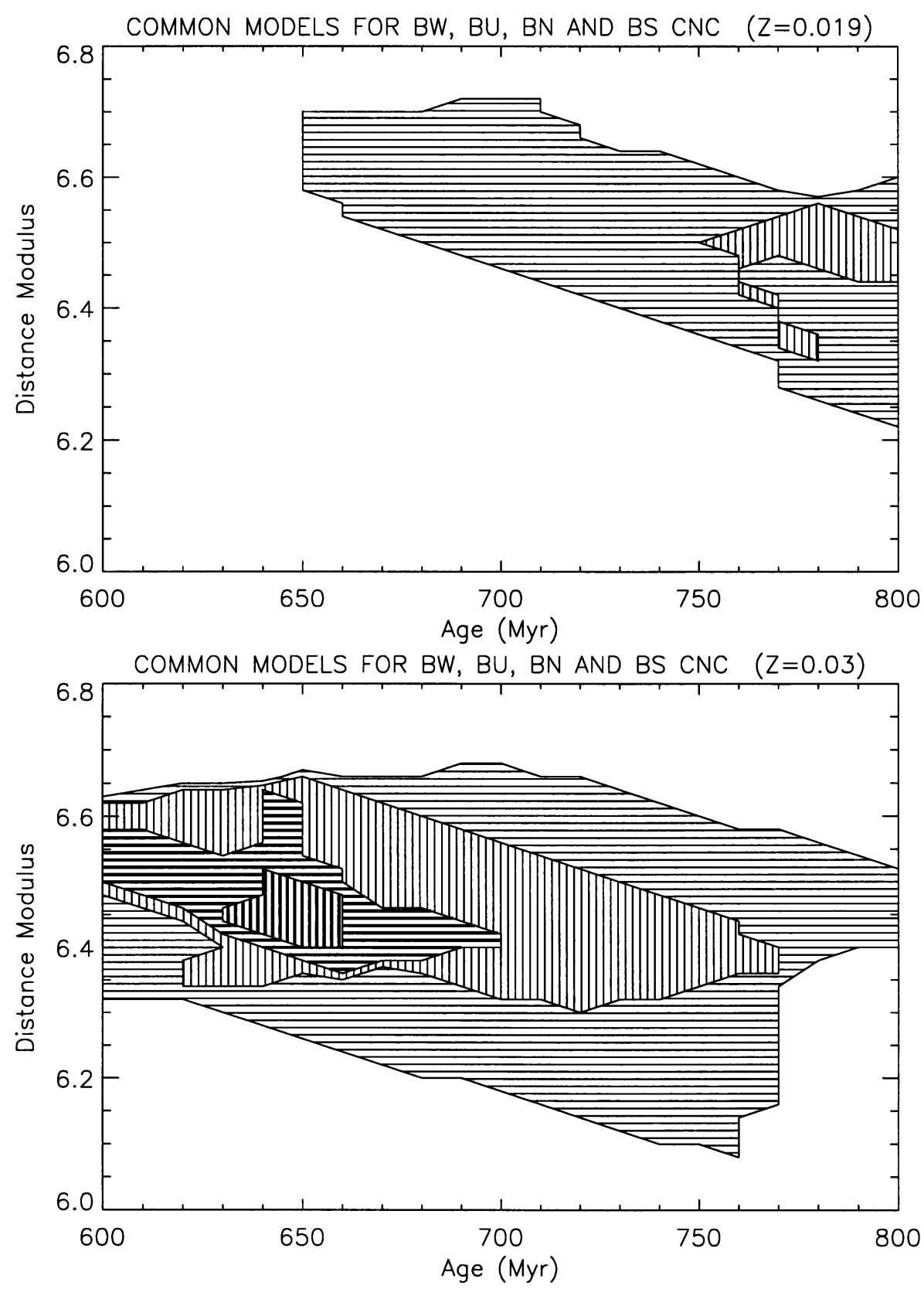

Figure 5. Valid models for 1 (horiz. thin), 2 (vert. thin), 3 (horiz. thick) and 4 stars (vert. thick) in Praesepe cluster with $Z=0.03$ for the only combination of potential pairs of radial modes with a common solution for all the stars. Common solutions with $Z=0.019$ for the same combination are also shown. Notice that, in this case, only two stars have common valid models. Blank spaces denote the models which cannot satisfy the observational and theoretical constraints on the cluster. 
A similar case appears for the cluster NGC 6134 observed by STACC, where $6 \delta$ Scuti stars were discovered. In this case, the lack (or even absence) of observational information about distance, metallicity or rotational velocity is responsible of the weak constraints which can be applied to the models. In Fig. 6, the frequency evolution versus the age for one of the variable stars and an attempt to identify their detected modes are shown. Despite of the uncertainties in the modelling, it can be seen that $g$-modes are necessary in order to explain the existence of some of the frequencies (Audard et al., 1995, 1997).

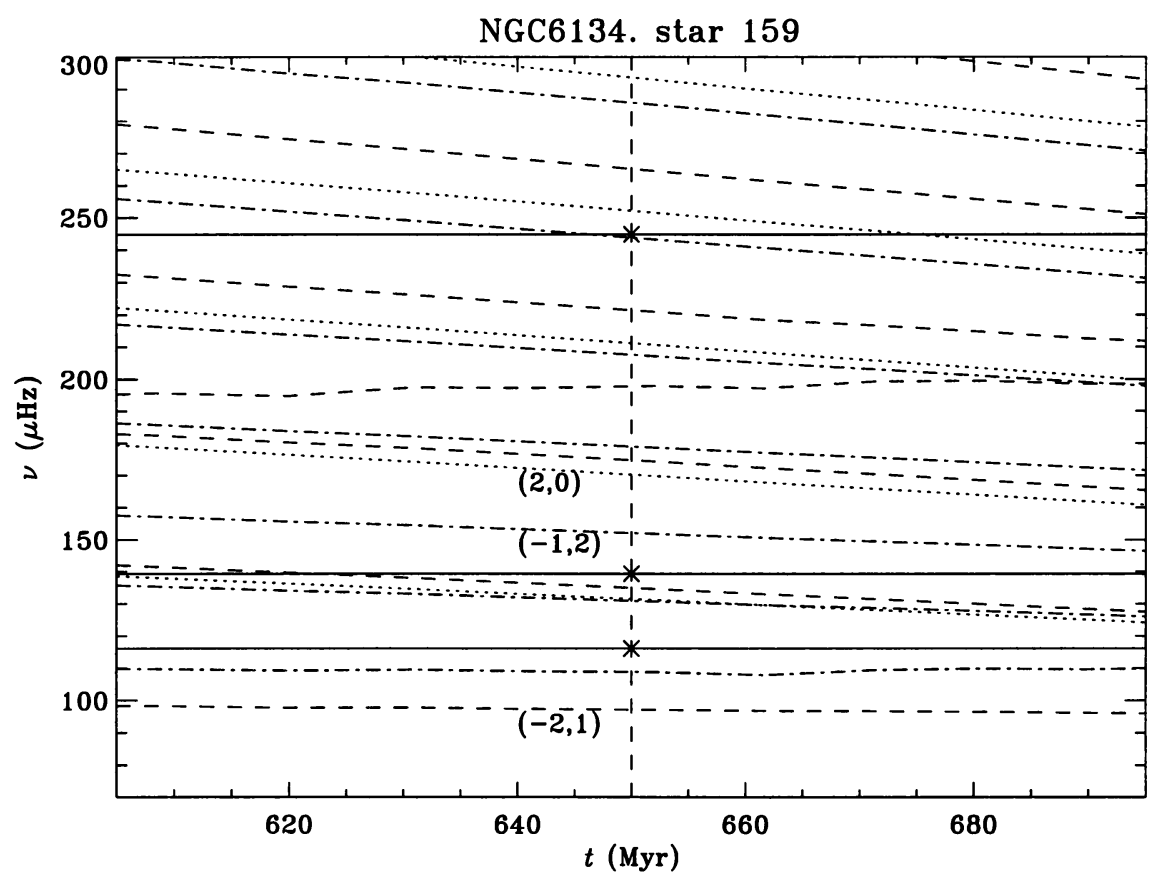

Figure 6. Variation with age of the theoretical frequencies for a model of $2.1 M_{\odot}$ in NGC 6134. The dashed vertical line gives the direct comparison of the frequencies detected in the star 159 (solid lines) at an age of $650 \mathrm{Myr}$. Dotted lines are for radial modes $l=0$, while dashed and dot-dashed lines are for modes of $l=1$ and 2 , respectively. The label $(n, l)$ is indicated for three modes. Despite of the non-existence of a unique identification, the possibility of having $g$-modes oscillating is clear in this star.

\section{Other achievements within ANTENA}

From the technical point of view, the realization of a improved version of the Four-Channel Stellar Photometer designed to perform rapid photometry 
(suitable for any kind of moderate amplitude pulsators: white dwarfs, roAp stars, $\delta$ Scuti or $\lambda$ Boo stars, etc.) in four channels simultaneously is the most representative achievement. This photometer has been successfully employed in the STEPHI 96 campaign. The Doppler Stellar Seismometer, designed to study solar-like oscillating stars with low amplitude $(1 \sigma \sim 10$ $\mathrm{cm} \mathrm{s}^{-1}$ ) is also in progress.

The mobility of researchers between the different member institutions of ANTENA for collaborating, either in the theoretical or the observational field, has been initiated fruitfully during these three years. At the same time, three new $\mathrm{PhD}$ students and two postdoctoral researchers have participated in these collaborations.

Finally, the possibility of reading these lines, after the network organization of the 181 IAU Symposium in Nice, dedicated to the memory of Philippe Delache, one of the head scientist of ANTENA died in October 1994, can be considered the culmination of most of the objectives contemplated by the members of the ANTENA project.

\section{References}

Audard, N., Kjeldsen, H. and Frandsen S. (1995) in R. S. Stobie and P. A. Whitelock (eds.), Astrophysical Applications of Stellar Pulsation, ASP Conf. Ser., Vol. 83, p. 325.

Audard, N., Kjeldsen, H. and Frandsen S. (1997) Astron. Astrophys. (to be submitted).

Belmonte, J. A., Michel, E., Álvarez, M., Jiang, S. Y., Chevreton, M., Auvergne, M., Liu, Y. Y., Goupil, M. J., Baglin, A. and Roca Cortés, T. (1994) Astron. Astrophys. 283, 121.

Frandsen, S. (1992) Delta Scuti News. 5, 12.

Frandsen, S., Balona, L. A., Viskum, M., Koen, C. and Kjeldsen, H. (1996) Astron. Astrophys. 308, 132.

Frandsen, S., Viskum, M., Hernández, M. M. and Belmonte, J. A. (1995) in R. S. Stobie and P. A. Whitelock (eds.), Astrophysical Applications of Stellar Pulsation, ASP Conf. Ser., Vol. 83, p. 327.

Hernández, M. M., Belmonte, J. A., Michel, E., Álvarez, M., Jiang, S. Y. and the STEPHI Network: (1996) Asteroseismology in Open Clusters: Praesepe, a Paradigm (this conference, poster book).

Hernández, M. M., Pérez Hernández, F., Michel, E., Belmonte, J. A., Goupil, M. J. and Lebreton, Y. (1996b) Observational constraints on Praesepe cluster by Asteroseismology (this conference, poster book).

Michel, E., Chevreton, M., Goupil, M. J., Belmonte, J. A., Jiang, S. Y., Álvarez, M., Suran M., Soufi F., Auvergne, M., Baglin, A., Liu, Y. Y., Vidal, I., Hernández, M. M. and Fu, J. N. (1995) in Fourth SOHO Workshop: Helioseismology, Pacific Grove, California, ESA SP-376, p. 533. 